\title{
Neuroendocrine dysfunction in patients recovering from subarachnoid hemorrhage
}

\author{
Vladimir Jovanovic ${ }^{1}$, Sandra Pekic ${ }^{2}$, Marko Stojanovic ${ }^{2}$, Goran Tasic ${ }^{1}$, \\ Branko Djurovic ${ }^{1}$, Ivan Soldatovic ${ }^{3}$, Mirjana Doknic ${ }^{2}$, Dragana Miljic ${ }^{2}$, Marina Djurovic ${ }^{2}$, \\ Milica Medic-Stojanoska ${ }^{4}$, Vera Popovic ${ }^{2}$
}

${ }^{1}$ Institute of Neurosurgery, ${ }^{2}$ Institute of Endocrinology, University Clinical Center, ${ }^{3}$ Institute for Medical Statistics and Health Research, Medical Faculty, Belgrade, ${ }^{4}$ Institute of Endocrinology, University of Novi Sad, Novi Sad, Serbia

\begin{abstract}
OBJECTIVE: Subarachnoid hemorrhage (SAH) is a recently identified risk factor for hypopituitarism, particularly growth hormone (GH) and corticotrophins deficiencies. The aim of our study was to identify possible predictor(s) for neuroendocrine dysfunction in SAH survivors. DESIGN: Pituitary function was evaluated in 93 patients (30 males, 63 females $)$, aged $48.0 \pm 1.1$ years (mean $\pm S E$ ), and with a Glasgow Outcome Scale score of 4.6 \pm 0.6 (mean $\pm S E$ ) more than one year following SAH. In the acute phase, SAH was complicated by vasospasm (VS) in 18 and by hydrocephalus (HDC) in 9 patients. Baseline serum values of Insulin Growth Factor 1 (IGF-I), cortisol, Thyroxine (T4), Thyroid Stimulating Hormone (TSH), Follicle Stimulating Hormone (FSH), Luteinizing Hormone (LH), testosterone (in males), estradiol (in females) and prolactin were determined. RESULTS: According to the results of baseline hormonal evaluation, 47 patients $(50.5 \%)$ had no hormonal abnormalities. Seven patients $(7.5 \%)$ had multiple pituitary hormone deficiencies: Four patients $(4.3 \%)$ had two (GH and cortisol), one patient had three (gonadal, adrenal and GH) and two patients had deficiency of all pituitary axes. Thirty-nine patients $(42 \%)$ had one abnormal axis (13 adrenal, 2 thyroid, 4 gonadal and 20 GH). None of the subjects was treated with desmopressin or exhibited symptomatic polyuria. The VS and HDC during the acute phase of SAH were related to abnormal pituitary status (VS with low IGF-I levels and HDC with low cortisol levels). CONCLUSION: Through a screening procedure, neuroendocrine dysfunction was identified in a substantial number of asymptomatic patients with previous SAH. Cerebral VS and HDC at the time of SAH emerged as risk factors possibly predicting development of pituitary dysfunction. Low basal levels of IGF 1 and cortisol may help in selecting patients requiring further evaluation of pituitary function.
\end{abstract}

Key words: Cerebral aneurysm, Hypopituitarism, Subarachnoid hemorrhage, Traumatic brain injury 


\section{INTRODUCTION}

Recent studies provide evidence for a high prevalence of neuroendocrine dysfunction in subarachnoid hemorrhage (SAH) survivors ranging from $27.5 \%$ to $55 \% .{ }^{1-6}$ Pituitary insufficiency in a patient after SAH caused by the rupture of an aneurysm of the anterior communicating artery was published in 1961 by Hoff et al. ${ }^{7}$ Kelly and colleagues reported pituitary insufficiency after SAH in two patients 3.5 and 13 months, respectively, after aneurysmal SAH. ${ }^{1}$ These patients presented with Growth Hormone Deficiency (GHD) diagnosed by Insulin Tolerance Test (ITT). Recent reports indicate a high prevalence of pituitary and hypothalamic abnormalities following SAH and TBI. ${ }^{1-6,8}$ It is still unclear whether the observed cognitive deficits are a consequence of the brain injury itself or are also related, at least in part, to the resulting anterior pituitary hormone deficiencies.

SAH shares some clinical features with traumatic brain injury (TBI), such as the loss of consciousness and amnesia. The pattern of cognitive and behavioral consequences of SAH are similar to those of TBI as well as of partial or complete pituitary insufficiency. ${ }^{9}$ Adult patients with GHD of non-SAH and non-TBI origin complain of attention and memory decline in spite of adequate adrenal, thyroid or sex hormone replacement therapy. ${ }^{10-12}$ The GH replacement therapy in these patients led to significant improvement of long- and short-term memory and cognitive functions. ${ }^{11}$

SAH accounts for about $5-10 \%$ of all strokes in people around 50 years of age, with the estimated annual incidence rate at between 10 to 25 cases per $100,000 .{ }^{13}$ The risk factors for aneurysmal SAH are hypertension and cigarette smoking. ${ }^{13} \mathrm{SAH}$ is fatal in more than half of the cases and serious disability is common among survivors. ${ }^{14}$ Modern management has reduced mortality and disability by about $30 \%$ compared with 30 years ago. Despite a good neurological outcome, patients surviving SAH frequently present with persistent cognitive and behavioral impairment. ${ }^{15,16}$ Over half showed clinically significant post-traumatic stress symptomatology at three months and $30 \%$ at nine months following SAH. ${ }^{17}$ Cognitive sequelae are most prominent in the domains of memory (visual short-term and verbal long-term), reaction-time task, concentration and nomination..$^{18}$ These consequences of SAH significantly affect the recovery and quality of life of patients and their families. Life-satisfaction and motivation are reduced, while emotional lability is increased. ${ }^{18}$ In the study of Bjeljac and colleagues, almost all patients presented with good neurological status [Glasgow Outcome Scale (GOS) score 4] one year after SAH, but only $30 \%$ exhibited no neuropsychological deficit. ${ }^{19}$

According to recently published recommendations for GH testing, patients who have survived traumatic brain injury and subarachnoid hemorrhage are included in the population who require evaluation of the hypothalamo-pituitary axes. ${ }^{20-22}$

We undertook the present pilot study to diagnose patients with increased risk for neuroendocrine dysfunction following SAH as well as to identify possible predictor(s) of neuroendocrine dysfunction.

\section{PATIENTS AND METHODS}

\section{Patients}

Long-term consequences of adult SAH survivors were studied by a team comprised of neurosurgeons and endocrinologists. The inclusion criteria for the study were: 1) history of aneurysmal SAH, 2) age 18 to 70 years at the time of testing, 3) time elapsed from the SAH between 1 and 10 years, and 4) good outcome after the hemorrhage, namely a GOS score of 4 to 5 . The exclusion criteria were: 1) history of severe depression (known to alter cortisol secretion) and 2) history of severe liver disease, kidney disease or uncontrolled diabetes mellitus.

Diagnosis of SAH was confirmed by computerized tomography (CT) scanning or lumbar puncture, and aneurysm location was determined by angiography (anterior communicating artery-ACoA, middle cerebral artery-MCA, internal carotid artery-ICA, vertebrobasilar artery-VBA). All patients were surgically treated. Hydrocephalus and cerebral vasospasm were considered as acute complications, while epilepsy was considered as a chronic complication of SAH.

Ninety-three SAH patients conforming to the inclusion and exclusion criteria were enrolled in the study. Body weight and height were measured and body mass index (BMI) was calculated. The previously 
known risk factors for SAH were recorded (arterial hypertension, smoking habits, diabetes mellitus). The study was approved by the hospital Ethics Committee and informed consent was obtained from all patients for the endocrine testing.

\section{Hormonal testing and assays}

Serum samples for insulin-like growth factor I (IGF-I), thyroxine (T4), TSH, FSH, LH, prolactin and cortisol determination were taken after an overnight fast, at 08:00. Serum total testosterone was determined in all males and serum estradiol in females. A menstrual history was taken in all female subjects. All samples were stored at $-80^{\circ} \mathrm{C}$ until assayed.

Hormones were measured by commercial kits: T4 by RIA (INEP, Zemun, Serbia). TSH by IRMA (INEP, Zemun, Serbia). PRL, LH and FSH by IRMA (Cis BioInternational, France). Cortisol, testosterone and estradiol were measured by RIA (Cis BioInternational, France). IGF-I was measured by chemiluminescent enzyme immunoassay with the Immulite Analyzer (Diagnostic Product Corporation, Los Angeles, CA, USA).

\section{Data analysis}

The standard reference ranges were used to discriminate abnormal from normal results of T4, TSH, basal cortisol, prolactin, FSH, LH, testosterone (in males) and estradiol (in females). Age- and genderspecific normal ranges were used for interpreting IGF-I levels. Menses within the past 42 days were considered normal.

Secondary hypocortisolism was diagnosed if morning serum cortisol level was less than $131 \mathrm{nmol} / 1$. TSH deficiency was defined as a low T4 level in the presence of an inappropriately low TSH level. In males, diagnosis of gonadotrophin deficiency was based on a low testosterone level in the presence of normal PRL and normal or low levels of gonadotrophins. In premenopausal women, secondary hypogonadism was diagnosed by low estradiol in the presence of normal PRL and normal or low levels of gonadotrophins. In postmenopausal women, secondary hypogonadism was considered if serum gonadotrophins were low for age. Levels of IGF-I lower than reference values for age- and gender of control subjects were considered abnormal.

\section{Statistical analysis}

Descriptive statistics are presented as mean value \pm SE. Statistical analysis was performed using the nonparametric Mann-Whitney test. The influence of age, BMI and GOS were analyzed with the Mann-Whitney test, while sex, localization of aneurysm, presence of cerebral vasospasm and/or hydrocephalus, epilepsy, arterial hypertension, diabetes mellitus and/or smoking habits were analyzed with the Chi-square test. Correlation between hormonal levels and various parameters in patients was analyzed using Spearman's correlation coefficient. The multiple logistic regression analysis with the Backward method was used to adjust for possible confounders on hormonal results. Analyses were performed using SPSS software (SPSS for Windows, release 10.0). $\mathrm{P}$ values less than 0.05 were regarded as indicating statistical significance.

\section{RESULTS}

\section{Study population}

Ninety-three SAH patients who fulfilled the inclusion and exclusion criteria were enrolled in the study. Subjects included 30 males and 63 females (23 premenopausal and 40 postmenopausal), aged $48.0 \pm 1.1$ years, with a mean BMI of $24.7 \pm 0.5 \mathrm{~kg} / \mathrm{m}^{2}$ and a mean GOS score of $4.6 \pm 0.6$. In our population with $\mathrm{SAH}$, more women (especially postmenopausal) than men were affected $(\mathrm{p}<0.001)$.

The patients were examined at least one year after the SAH (mean \pm SE: $1.8 \pm 0.2$ years). The age at occurrence of $\mathrm{SAH}$ was (mean $\pm \mathrm{SE}$ ) $46.2 \pm 1.1$ years.

The location of the aneurysm was at the internal carotid artery (ICA) in $32(34.4 \%)$, at the middle cerebral artery (MCA) in $30(32.3 \%)$, at the anterior communicating artery (ACoA) in $22(23.7 \%)$ and at the vertebrobasilar arteries in $9(9.7 \%)$ patients. Cerebral vasospasm (VS) during the acute phase of SAH was registered in $18(19.4 \%)$ patients, most frequently in patients with aneurysm of the ICA (10 of 32 cases). Hydrocephalus (HDC) during the acute phase of SAH was registered in $9(9.7 \%)$ patients, most frequently in postmenopausal women. Two patients had both complications, cerebral VS and HDC, during the acute phase of SAH. Epilepsy, as a chronic complication of SAH, was diagnosed in four patients. 
Arterial hypertension at the time of hemorrhage was diagnosed in $49(52.7 \%)$ patients, most frequently in postmenopausal women. Five patients were diabetic at the time of acute hemorrhage and $57(61.3 \%)$ were active smokers.

\section{Results of hormonal evaluation}

The prevalence of neuroendocrine dysfunction in SAH patients is presented in Figures 1 and 2. Overall, 47 patients $(50.5 \%$ ) had no abnormalities. Seven patients $(7.5 \%)$ had multiple pituitary hormonal deficiencies (Figure 1): Four patients (4.3\%) had dual abnormalities (GH and adrenal axis), one female patient had three abnormal axes (gonadal,

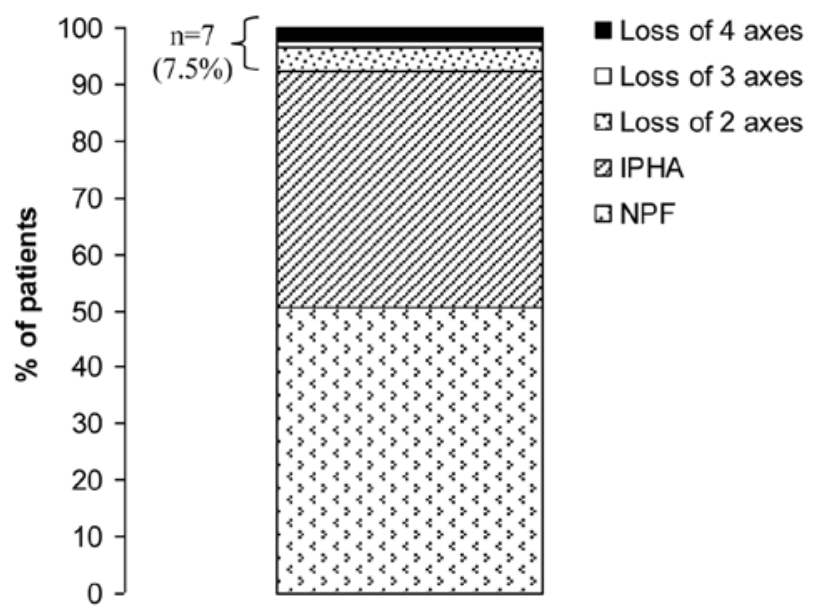

Figure 1. Prevalence of normal pituitary function (NPF; $n=47$, $50.5 \%$ ), isolated pituitary hormone abnormality (IPHA; $\mathrm{n}=39$, $42 \%)$ or loss of two, three and four pituitary axes $(n=7,7.5 \%)$ in patients tested more than one year after aneurysmal SAH.

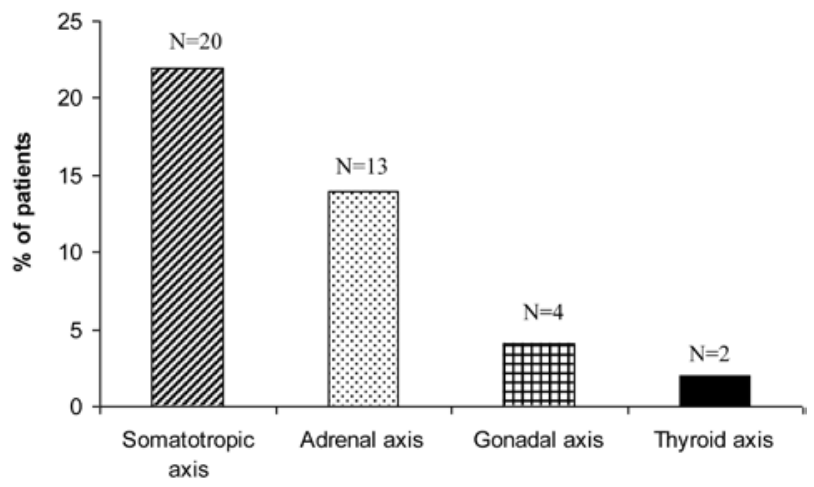

Figure 2. Distribution of single pituitary deficit in 39 patients ( $42 \%$ of all SAH patients) tested more than one year after aneurysmal SAH. adrenal and $\mathrm{GH}$ ) and two female patients had all four pituitary axes affected. Thirty-nine patients (42\%) had a single abnormal axis (13 adrenal, 2 thyroid, 4 gonadal and $20 \mathrm{GH}$; Figure 2). None of the subjects had been treated with desmopressin or exhibited symptomatic polyuria.

Clinical characteristics of patients with normal pituitary function (NPF), those with isolated pituitary hormone abnormality (IPHA) and those with multiple pituitary hormone abnormalities (MPHA) are presented in Table 1. Patients with MPHA and IPHA had increased incidence of cerebral VS compared with those with normal pituitary function $(\mathrm{p}<0.001)$. Patients with IPHA had a lower GOS compared with patients with normal pituitary function $(p=0.020)$.

\section{Somatotropic axis}

IGF-I levels were below normal values for ageand sex-matched healthy subjects in $27 \mathrm{SAH}$ patients (29.0\%), indicating the need for GH provocative testing to confirm the diagnosis of GHD. In 20 patients GHD was an isolated pituitary hormone abnormality (IPHA; Figure 2), and in seven low IGF-I levels were combined with other pituitary hormone abnormali-

Table 1. Clinical characteristics of patients with normal pituitary function (NPF), those with isolated pituitary hormone abnormality (IPHA) and those with multiple pituitary hormone abnormalities (MPHA)

\begin{tabular}{lccc}
\hline & NPF & IPHA & MPHA \\
\hline $\mathrm{N}$ & 47 & 39 & 7 \\
Sex (male/female) & $17 / 30$ & $11 / 28$ & $2 / 5$ \\
Age (years) & $49.3 \pm 1.4$ & $45.5 \pm 1.7$ & $54.8 \pm 1.7^{\mathrm{d}}$ \\
BMI (kg/m ${ }^{2}$ ) & $23.9 \pm 0.6$ & $25.6 \pm 0.7^{\mathrm{b}}$ & $25.5 \pm 2.3^{\mathrm{b}}$ \\
Time since SAH (yrs) & $1.9 \pm 0.2$ & $1.7 \pm 0.2$ & $1.8 \pm 0.4$ \\
Vasospasm (yes/no) & $2 / 45$ & $13 / 26^{\mathrm{a}}$ & $3 / 4^{\mathrm{a}}$ \\
Hydrocephalus (yes/no) & $2 / 45$ & $5 / 34$ & $2 / 5$ \\
Epilepsy (yes/no) & $4 / 43$ & $0 / 39$ & $0 / 7$ \\
GOS score & $4.7 \pm 0.1$ & $4.4 \pm 0.1^{\mathrm{c}}$ & $4.6 \pm 0.2$ \\
Hypertension (yes/no) & $24 / 23$ & $23 / 16$ & $2 / 5$ \\
Diabetes mellitus (yes/no) & $4 / 43$ & $1 / 38$ & $0 / 7$ \\
Smoking (yes/no) & $29 / 18$ & $25 / 14$ & $3 / 4$ \\
\hline
\end{tabular}

${ }^{\mathrm{a} p}<<0.001$ IPHA and MPHA vs. patients with NPF; ${ }^{\mathrm{b}} \mathrm{p}<0.05$ IPHA and MPHA vs. patients with NPF; ${ }^{c} p<0.05$ IPHA vs. patients with NPF; ${ }^{\mathrm{p}}<0.05$ MPHA vs. IPHA Descriptive statistics for continuous variables are presented as mean value \pm SE. 
ties. In four patients, low IGF-I level was associated with low morning cortisol levels (two axes affected). In one female, low IGF-I level was associated with low morning cortisol and estradiol/gonadotrophin levels (three axes affected), while in two females all four pituitary axes were affected.

The IGF-I level in patients with cerebral VS during the acute phase of SAH was significantly lower compared with patients without VS $(74.3 \pm 18.5 \mathrm{ng} / \mathrm{ml}$ vs. $154.5 \pm 12.0 \mathrm{ng} / \mathrm{ml} ; \mathrm{p}<0.001$; Figure $3 \mathrm{a})$. The patients who had VS during the acute phase of SAH had 13.5-higher risk for low IGF-I level and possible GHD in the chronic phase $(\mathrm{OR}=13.538 ; \mathrm{p}<0.001$; 95\% CI=3.311-55.361; Table 2a). The IGF-I level was not significantly different in patients with and without HDC during the acute phase of SAH $(p=0.158)$.

\section{Adrenal axis}

Basal morning cortisol levels were below the lower limit of normal range $(<131 \mathrm{nmol} / \mathrm{l})$ in 20 of 93 patients $(21.5 \%)$. In 13 patients, low cortisol levels presented as an isolated hormone deficiency (Figure 2). The remaining seven patients presented additionally low IGF-I levels (in four patients), low IGF-I and gonadotrophins deficiency (in one postmenopausal woman) and in two postmenopausal women low cortisol level was associated with impairment of all other pituitary axes.

The cortisol level in the patients with cerebral VS during the acute phase of SAH was significantly lower compared with patients without VS $(230.6 \pm 25.4 \mathrm{nmol} / 1$ vs. $310.7 \pm 16.1 \mathrm{nmol} / 1 ; \mathrm{p}<0.05$; Figure $3 \mathrm{a}$ ). The patients who had VS during the acute phase of SAH had 3.8fold increased probability (not reaching statistical significance, defined by a p of less than 0.05 , but still with a notable $p$ of 0.073 ) of having low cortisol level and possible secondary hypocortisolism in the chronic phase $(\mathrm{OR}=3.794 ; \mathrm{p}=0.073 ; 95 \% \mathrm{CI}=0.881-16.337)$. The presence of HDC during the acute phase of SAH increased the risk for low cortisol level more than 6-fold $(\mathrm{OR}=6.346 ; \mathrm{p}=0.012 ; 95 \% \mathrm{CI}=1.499-26.867$; Table $2 b$ ).

\section{Gonadal axis}

Total testosterone level was below the normal range in two $(6.7 \%)$ of 30 males, presenting in both as an isolated hormone deficiency. There was no correlation between testosterone levels and history of cerebral VS or HDC.

Of 63 female subjects, $40(63.5 \%)$ were classified as postmenopausal by menstrual history. Of postmenopausal subjects high gonadotrophins were confirmed in 35 , while five had inappropriately low gonadotrophins (secondary hypogonadism). In one patient secondary hypogonadism was combined with low basal cortisol and IGF-I levels, while in two postmenopausal women all pituitary axes were affected. A further 23 females had regular menstrual cycles.

\section{Thyroid axis}

T4 level was below the lower limit of the normal range in four of 93 patients $(4.3 \%)$ and was associated with low-normal TSH levels (secondary hypothyroidism). In two patients secondary hypothyroidism was an IPHA, while in two all pituitary axes were affected.

\section{Lactotropic axis}

Prolactin levels were mildly elevated in four patients, combined in two cases with low IGF-I levels and in one female patient with impairment of all pituitary axes. There was no correlation between prolactin levels and history of cerebral VS or HDC.

\section{Acute complications of SAH (cerebral vasospasm and hydrocephalus)}

Patients with cerebral VS had a lower GOS score compared with patients without cerebral VS during the acute phase of SAH ( $<<0.001$, Figure 3b). IGF-I and cortisol levels were significantly lower in patients with a history of VS compared with those without VS (Figure 3a). Two patients had both complications during the acute phase of SAH. One of them had three pituitary axes affected (low IGF-I, low cortisol and low gonadotropin levels), while the other patient had low cortisol levels.

\section{Localization of aneurysm and endocrinological evaluation}

Results of hormonal analysis in patients divided into four groups according to the localization of aneurysm are presented in Table 3. Patients with aneurysm of ICA had a lower IGF-I level compared with patients with aneurysm of MCA $(p<0.05)$. Male patients with aneurysm of VB had lower testosterone 
Table 2a. The influence of confounding factors on IGF-I level (multiple logistic regression analysis with Backward method)

\begin{tabular}{|c|c|c|c|c|c|}
\hline \multirow{2}{*}{\multicolumn{2}{|c|}{$\begin{array}{l}\text { Logistic regression } \\
\text { Method: Backward }\end{array}$}} & \multirow[b]{2}{*}{ p value } & \multirow[b]{2}{*}{ OR } & \multicolumn{2}{|c|}{ 95.0\% CI for OR } \\
\hline & & & & Lower & Upper \\
\hline \multirow[t]{9}{*}{ First step } & localization & 0.958 & 1.016 & 0.552 & 1.871 \\
\hline & vasospasm & 0.002 & 11.155 & 2.409 & 51.667 \\
\hline & hydrocephalus & 0.338 & 2.511 & 0.382 & 16.489 \\
\hline & hypertension & 0.947 & 0.961 & 0.293 & 3.145 \\
\hline & diabetes mellitus & 0.580 & 0.457 & 0.028 & 7.343 \\
\hline & smoking & 0.858 & 1.125 & 0.309 & 4.091 \\
\hline & epilepsy & 0.999 & 0.000 & 0.000 & . \\
\hline & GOS score & 0.493 & 0.714 & 0.273 & 1.868 \\
\hline & gender & 0.651 & 1.371 & 0.349 & 5.382 \\
\hline Final step & vasospasm & 0.000 & 13.538 & 3.311 & 55.361 \\
\hline
\end{tabular}

OR - odd ratio; CI - confidence interval

Table 2b. The influence of confounding factors on cortisol level (multiple logistic regression analysis with Backward method)

\begin{tabular}{|c|c|c|c|c|c|}
\hline \multirow{2}{*}{\multicolumn{2}{|c|}{$\begin{array}{l}\text { Logistic regression } \\
\text { Method: Backward }\end{array}$}} & \multirow[b]{2}{*}{ p value } & \multirow[b]{2}{*}{ OR } & \multicolumn{2}{|c|}{ 95.0\% CI for OR } \\
\hline & & & & Lower & Upper \\
\hline \multirow[t]{7}{*}{ First step } & localization & 0.472 & 0.784 & 0.403 & 1.522 \\
\hline & vasospasm & 0.073 & 3.794 & 0.881 & 16.337 \\
\hline & hydrocephalus & 0.010 & 9.021 & 1.688 & 48.219 \\
\hline & hypertension & 0.505 & 1.513 & 0.447 & 5.117 \\
\hline & diabetes mellitus & 0.185 & 0.445 & 0.134 & 1.474 \\
\hline & smoking & 0.507 & 1.403 & 0.516 & 3.815 \\
\hline & epilepsy & 0.298 & 0.487 & 0.126 & 1.884 \\
\hline Final step & hydrocephalus & 0.012 & 6.346 & 1.499 & 26.867 \\
\hline
\end{tabular}

OR - odd ratio; CI - confidence interval

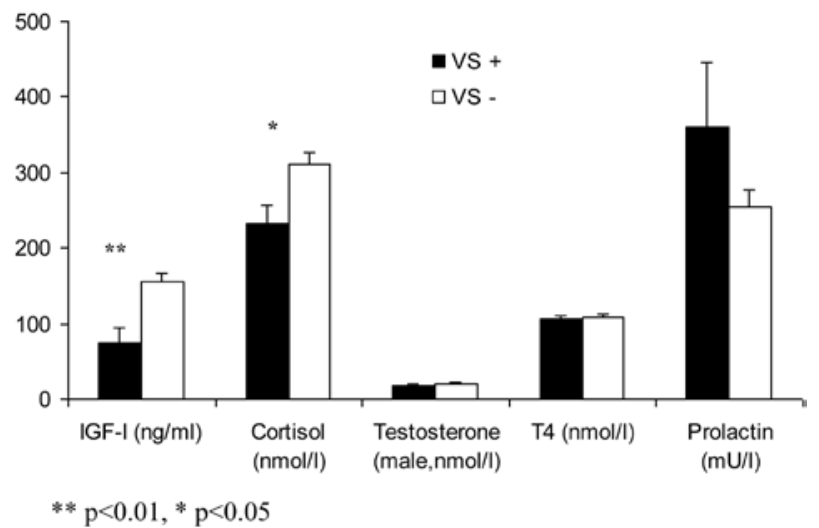

FIGURE 3a. Basal hormonal levels in patients with (VS+) and without vasospasm (VS-) during the acute phase of SAH.

level compared with patients with aneurysm of ICA $(\mathrm{p}<0.05)$.

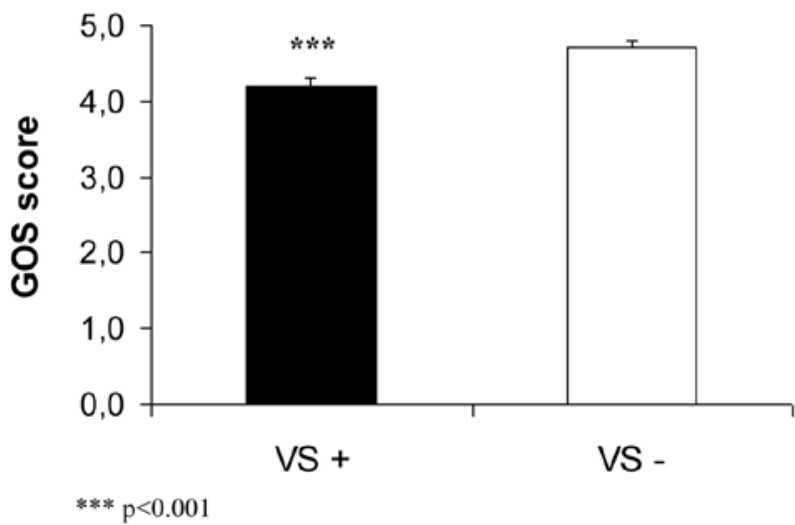

FIGURE 3b. Glasgow Outcome Scale (GOS) score in patients with (VS+) and without vasospasm (VS-) during the acute phase of SAH.

\section{DISCUSSION}

The results of this study showed that almost half of the patients with SAH presented some degree of 
Table 3. Hormonal analysis in patients with various localizations of the aneurysm

\begin{tabular}{lllll}
\hline & \multicolumn{1}{c}{ ICA } & \multicolumn{1}{c}{ MCA } & ACoA & VB \\
\hline $\mathrm{N}$ & $32(34.4 \%)$ & $30(32.3 \%)$ & $22(23.7 \%)$ & $9(9.7 \%)$ \\
T4 (nmol/l) & $113.8 \pm 4.5$ & $106.1 \pm 4.4$ & $102.9 \pm 5.9$ & $106.2 \pm 8.6$ \\
Testosterone (male, nmol/l) & $21.0 \pm 1.7$ & $18.2 \pm 5.2$ & $19.6 \pm 2.8$ & $13.8 \pm 0.9 \mathrm{~b}$ \\
Cortisol (nmol/l) & $276.1 \pm 20.7$ & $303.5 \pm 29.3$ & $297.2 \pm 28.5$ & $331.9 \pm 45.1$ \\
Prolactin (mU/l) & $267.1 \pm 23.6$ & $247.6 \pm 23.6$ & $356.6 \pm 68.5$ & $202.6 \pm 30.0$ \\
IGF-I (ng/ml) & $109.5 \pm 14.0 \mathrm{a}$ & $162.9 \pm 19.4$ & $147.4 \pm 28.7$ & $126.2 \pm 30.4$ \\
\hline
\end{tabular}

Descriptive statistics for continuous variables are presented as mean value $\pm \mathrm{SE}$ ${ }^{\mathrm{a}} \mathrm{p}<0.05$ ICA vs MCA, ${ }^{\mathrm{b}} \mathrm{p}<0.05$ ICA vs VB

ICA: Internal Carotid Artery, MCA: Middle Cerebral Artery, ACoA: Anterior Communicating Artery, VB: Vertebrobasilar Artery

hypopituitarism. Thirty-nine patients $(42 \%)$ had one abnormal axis $(20 \mathrm{GH}, 13$ adrenal, 4 gonadal and 2 thyroid) and seven (7.6\%) had multiple abnormal axes. Of the seven patients with multiple abnormal axes, four had two abnormalities (GH and adrenal), one had three $(\mathrm{GH}$, adrenal and gonadal) and two all four pituitary axes affected. The most vulnerable hypothalamic-pituitary axes were somatotropic and adrenal, while gonadal and thyroid axes were less frequently affected. Disturbances of the posterior pituitary were not encountered. Overall, half of the patients investigated one or more years after SAH presented with insufficiency of one or more anterior pituitary axes. The history of cerebral vasospasm and hydrocephalus during acute $\mathrm{SAH}$ was predictive for neuroendocrine dysfunction in the chronic phase.

Hypopituitarism may be the acute or chronic consequence of TBI and SAH. ${ }^{1-8,12}$ The first large multicenter study on SAH patients was carried out by Aimaretti and colleagues who investigated 40 patients three months after SAH and diagnosed a variable degree of hypopituitarism in $37.5 \%$ of the patients. ${ }^{5}$ In contrast to TBI, pituitary deficits after $\mathrm{SAH}$ are more often isolated than multiple and panhypopituitarism has not been reported. The most frequently affected pituitary axes in SAH survivors are the somatotropic axis and adrenal axis, as in our study. ${ }^{1-6}$ Secondary hypogonadism was diagnosed in $13 \%$ of patients, while secondary hypothyroidism was less frequent, diagnosed in up to $7.5 \%$ of SAH survivors. ${ }^{5}$ The diversity in the results of various studies following SAH may be attributed to differences in the methods used in the evaluation of neuroendocrine function, particularly concerning the somatotropic and adrenal axes. In TBI and SAH patients the most frequently affected axis is the somatotropic. ${ }^{1-8}$ Various tests have been used to diagnose GH deficiency, such as IGF-I determination and stimulatory tests (ITT and GHRH+arginine test). ${ }^{1-8}$ The role of IGF-I in the diagnosis of adult GHD has been confusing due to the complexity of the regulation of IGF-I synthesis. ${ }^{23}$ A considerable overlap exists in IGF-I levels between normal subjects and patients with GHD. ${ }^{24}$ Serum IGF-I levels might be normal even in patients with total anterior hypopituitarism. However, despite the low diagnostic sensitivity of this parameter, very low levels of total IGF-I can be considered definitive evidence of severe GHD in a remarkable percentage of hypopituitary patients who could therefore skip provocative testing of GH secretion. ${ }^{25}$ In our study, serum IGF-I measurements in adult patients investigated for possible GHD were compared with decade-based normative data and levels of IGF-I lower than the normative data were considered abnormal. Dimopoulou and colleagues found GHD in $37 \%$ of SAH patients based on low IGF-I level. ${ }^{3}$ The most common alteration in their and our study was a diminished secretion of $\mathrm{GH}$, as reflected by the finding of low IGF-I levels. We found IGF-I levels below decade-based normative data in $29 \%$ of SAH patients and identified the cerebral vasospasm during the acute phase of SAH as a risk factor for low IGF-I level in these patients. The studies in which a stimulatory test for $\mathrm{GH}$ was used revealed that 20 $22 \%$ of SAH patients were GHD. ${ }^{2,4}$ In our patients low IGF-I levels, particularly in those with IPHA, 
underlines the need for $\mathrm{GH}$ provocative testing for confirmation of GHD.

The second most affected axis was the adrenal axis. Aimaretti and colleagues restricted the diagnosis of secondary hypocortisolism to the basal morning cortisol level less than $220 \mathrm{nmol} / \mathrm{l}$ and the low cortisol level in 24-hour urine. ${ }^{2,5}$ In their study, secondary hypocortisolism was found in only $2.5 \%$ of SAH patients tested three months after the acute event. In another study, low-dose ACTH testing revealed secondary hypocortisolism in $10 \%$ of SAH patients tested 12-24 months after SAH. ${ }^{3}$ In addition, when ITT was used, secondary hypocortisolism was diagnosed in $40 \%$ of SAH patients tested $12-72$ months after SAH. ${ }^{4}$ Schneider and colleagues reviewed the literature regarding SAH and pooled prevalences of hypopituitarism in the chronic phase of SAH. They found that $20.5 \%$ of SAH patients had secondary hypocortisolism. ${ }^{5}$ These data are in accordance with our results. We identified $21.5 \%$ of SAH patients with low morning cortisol level. In published studies there is a high incidence of isolated corticotrophins deficiency following SAH. ${ }^{6}$ These findings correspond to the neuropathological findings of selective hemorrhages into the paraventricular nuclei of the hypothalamus, indicating hypothalamic rather than pituitary dysfunction. ${ }^{26}$ The findings of frequent hypocortisolism after SAH are in contrast to findings of neuroendocrine dysfunction following TBI in which $\mathrm{LH} / \mathrm{FSH}$ and $\mathrm{GH}$ deficiencies are significantly more common than ACTH deficiency, which is also more common than TSH deficiency ${ }^{6,8}$

Hypopituitarism is often associated with mild hyperprolactinemia resulting from neurosecretory dysfunction. In our study in three SAH patients hyperprolactinemia was associated with low IGF-I levels. Other deficits, e.g. gonadal and thyroid deficits, are less frequently observed in all published studies.

Systematic neuropathological investigations of the hypothalamo-pituitary axis after aneurysmal SAH have not been conducted so far. There are scarce pathological data about possible pathophysiological mechanisms of neuroendocrine dysfunction following SAH. Pathophysiological changes during the acute phase of SAH include the rise in intracranial pressure, cerebral vasospasm and impairment of cerebral perfusion and focal or global brain edema. Furthermore, SAH can be complicated by intracerebral and intraventricular hemorrhage and acute hydrocephalus. Hemorrhage into the pituitary has frequently been observed after the rupture of an intracranial aneurysm. ${ }^{27}$ Some authors described confluent hemorrhages which destroyed the pituitary gland and a hemorrhagic focus in the infundibulum in cases of increased intracranial pressure. ${ }^{28}$ Neuropathological studies found evidences of morphological hypothalamic damage (hemorrhagic and ischemic changes) in $68 \%$ of patients who died shortly after the rupture of an intracranial aneurysm. ${ }^{26}$ These microhemorrhages were selectively located in the paraventricular and supraoptic nuclei in most cases. The author postulated that this specific location of microhemorrhages was the consequence of the increased pressure in the chiasmatic cistern and a temporary obstruction of venous drainage in these nuclei. Ruptured aneurysms of the anterior communicating artery and the posterior communicating artery were more often associated with hypothalamic lesions than aneurysms of other locations. Thus, possible pathophysiological mechanisms of neuroendocrine dysfunction following SAH include: direct effect of hemorrhage, mechanical (an abrupt rise of intracranial pressure during the acute $\mathrm{SAH}$ ), toxic, inflammatory and vascular (ischemia due to vasospasm).

The high percentage of disturbed somatotroph and adrenal axes in our study may represent an overestimation as the diagnosis is based on low basal cortisol and low IGF-I levels. We are aware that a number of patients with mild reduction of serum cortisol levels and IGF-I levels would respond normally to ITT, but this test is contraindicated in patients after recent brain injury. ${ }^{29}$ It must be stressed, however, that adult GHD would be strongly predicted by very low IGF-I levels. ${ }^{23,26,30}$

In conclusion, neuroendocrine dysfunction following aneurysmal subarachnoid hemorrhage may occur at a much higher prevalence than previously suspected. High incidence of corticotroph and somatotroph dysfunction was observed in this study, with cerebral vasospasm and hydrocephalus emerging as the risk factors for dysfunction of the hypothalamopituitary axes. 
There is a need for the formulation of an appropriate screening program for patients with brain injuries, which will help in the identification of subjects at high risk of developing an unrecognized hypopituitarism, a potentially serious but treatable complication of SAH. The presence of VS and HDC are factors that could be used for the selection of subjects with SAH who require further evaluation of pituitary function. Certainly, neurosurgeons, neurologists and endocrinologists should be aware of the considerable risk of pituitary dysfunction following SAH.

\section{REFERENCES}

1. Kelly DF, Gonzalo IT, Cohan P, Berman N, Swerdloff R, Wang C, 2000 Hypopituitarism following traumatic brain injury and aneurysmal subarachnoid hemorrhage: a preliminary report. J Neurosurg 93: 743-752.

2. Aimaretti G, Ambrosi B, Di Somma C, et al, 2004 Traumatic brain injury and subarachnoid haemorrhage are conditions at high risk for hypopituitarism: screening study at 3 months after the brain injury. Clin Endocrinol (Oxf) 61: 320-326.

3. Dimopoulou I, Kouyialis AT, Tzanella M, et al, 2004 High incidence of neuroendocrine dysfunction in longterm survivors of aneurysmal subarachnoid hemorrhage. Stroke 35: 2884-2889.

4. Kreitschmann-Andermahr I, Hoff C, Saller B, et al, 2004 Prevalence of pituitary deficiency in patients after aneurysmal subarachnoid hemorrhage. J Clin Endocrinol Metab 89: 4986-4992.

5. Aimaretti G, Ambrosio MR, Di Somma C, et al, 2005 Residual pituitary function after brain injury-induced hypopituitarism: a prospective 12-month study. J Clin Endocrinol Metab 90: 6085-6092.

6. Schneider HJ, Kreitschmann-Andermahr I, Ghigo E, Stalla GK, Agha A, 2007 Hypothalamopituitary dysfunction following traumatic brain injury and aneurysmal subarachnoid hemorrhage. JAMA 298: 1429-1438.

7. Hoff WV, Hornabrook RW, Marks V, 1961 Hypopituitarism associated with intracranial anerysms. Br Med J 5261: 1190-1194.

8. Lieberman SA, Oberoi AL, Gilkison CR, Masel BE, Urban RJ, 2001 Prevalence of neuroendocrine dysfunction in patients recovering from traumatic brain injury. J Clin Endocrinol Metab 86: 2752-2756.

9. Vance ML, 1994 Hypopituitarism. N Engl J Med 330: 1651-1662.

10. Deijen J, de Boer H, Blok GJ, van der Veen EA, 1996 Cognitive impairments and mood disturbances in growth hormone deficient men. Psychoneuroendocrinology 21: 313-322.

11. Bulow B, Hagman L, Orbaek P, Osterberg K, Erfurth
EM, 2002 High incidence of mental disorders, reduced mental well-being and cognitive function in hypopituitary women with GH deficiency treated for pituitary disease. Clin Endocrinol (Oxf) 56: 183-193.

12. Popovic V, Pekic S, Pavlovic D, et al, 2004 Hypopituitarism as a consequence of traumatic brain injury (TBI) and its possible relation with cognitive and mental distress. J Endocrinol Invest 27: 1048-1054.

13. Leon-Carrion J, Dominguez-Morales M del R, Barroso y Martin JM, Murillo-Cabezas F, 2005 Epidemiology of traumatic brain injury and subarachnoid hemorrhage. Pituitary 8: 197-202.

14. Van Gijn J, Rinkel GJE, 2001 Subarachnoid haemorrhage: diagnosis, causes and management. Brain 124: 249-278.

15. Ogden JA, Mee EW, Henning M, 1993 A prospective study of impairment of cognition and memory and recovery after subarachnoid hemorrhage. Neurosurgery 33: 572-586.

16. Mayer SA, Kreiter KT, Copeland D, et al, 2002 Global and domain-specific cognitive impairment and outcome after subarachnoid hemorrhage. Neurology 10: 1750-1758.

17. Powell J, Kitchen N, Heslin J, Greenwood R, 2002 Psychosocial outcomes at three and nine months after good neurological recovery from aneurysmal subarachnoid haemorrhage: predictors and prognosis. J Neurol Neurosurg Psychiatry 75: 1119-1124.

18. Hutter BO, Gilsbach JM, Kreitschmann I, 1995 Quality of life and cognitive deficits after subarachnoid haemorrhage. Br J Neurosurg 9: 465-475.

19. Bjeljac M, Keller E, Regard M, Yonekawa Y, 2002 Neurological and neuropsychological outcome after SAH. Acta Neurochir Suppl 82: 83-85.

20. Ho KKY, 2007 Consensus guidelines for the diagnosis and treatment of adults with GH deficiency II: a statement of the GH Research Society in association with the European Society for Pediatric Endocrinology, Lawson Wilkins Society, European Society of Endocrinology, Japan Endocrine Society, and Endocrine Society of Australia. Eur J Endocrinol 157: 695-700.

21. Casanueva FF, Catro AI, Micic D, Kelestimur F, Dieguez C, 2009 New guidelines for the diagnosis of growth hormone deficiency in adults. Horm Res 71: Suppl 1: 112-115.

22. Sesmilo G, Halperin I, Puig-Domingo M, 2007 Endocrine evaluation of patients after brain injury: what else is needed to define specific clinical recommendation? Hormones (Athens) 6: 132-137.

23. Mukherjee A, Shalet SM, 2009 The value of IGF1 estimation in adults with GH deficiency. Eur J Endocrinol 161: S33-S39.

24. Hoffman DM, O’Sullivan AJ, Baxter RC, Ho KK, 1994 Diagnosis of growth hormone-deficiency in adults. Lancet 343: 1064-1068.

25. Aimaretti G, Corneli G, Baldelli R, et al, 2003 Diag- 
nostic reliability of a single IGF-I measurement in 237 adults with total anterior hypopituitarism and severe GH deficiency. Clin Endocrinol 59: 56-61.

26. Crompton MR, 1963 Hypothalamic lesions following the rupture of cerebral berry aneurysms. Brain 86 : $301-$ 314.

27. Kornblum RN, Fisher RS, 1969 Pituitary lesions in craniocerebral injuries. Arch Pathol 88: 242-248.

28. Rap Z, Zaremba J, 1971 Pathomechanism of secondary morphological lesions in the hypothalamo-hypophyseal system in cases of increased intracranial pressure. Pol Med J 10: 188-207.

29. Lamberts SWJ, de Herder WW, van der Lely AJ, 1998 Pituitary insufficiency. Lancet 352: 127-134.

30. Hartman ML, Crowe BJ, Biller BM, Ho KK, Clemmons DR, Chipman JJ, HyposCCS Advisory Board \& US HypoCCS Study Group, 2002 Which patients do not require a $\mathrm{GH}$ stimulation test for the diagnosis of adult GH deficiency? J Clinical Endocrinol Metab 87: 477-485. 